\title{
Estimated number of prevalent cases of metastatic bone disease in the US adult population
}

This article was published in the following Dove Press journal:

Clinical Epidemiology

5 April 2012

Number of times this article has been viewed

\author{
Shuling $\mathrm{Li}^{\prime}$ \\ Yi Peng' \\ Eric D Weinhandl' \\ Anne H Blaes ${ }^{2}$ \\ Karynsa Cetin ${ }^{3}$ \\ Victoria M Chia ${ }^{3}$ \\ Scott Stryker ${ }^{3}$ \\ Joseph J Pinzone ${ }^{4}$ \\ John F Acquavella ${ }^{3}$ \\ Thomas J Arneson' \\ 'Chronic Disease Research Group, \\ Minneapolis Medical Research \\ Foundation, Minneapolis, MN, USA; \\ ${ }^{2}$ Division of Hematology, Oncology \\ and Transplantation, University of \\ Minnesota, Minneapolis, MN, USA; \\ ${ }^{3}$ Center for Observational Research, \\ ${ }^{4}$ Global Development, Amgen, \\ Thousand Oaks, CA, USA
}

Correspondence: Thomas J Arneson Chronic Disease Research Group, Minneapolis Medical Research Foundation, 914 South 8th Street, Suite S-406, Minneapolis, MN 55404, USA

Tel + I 6123475355

Fax + I 6123475980

Email tarneson@cdrg.org
Background: The prevalence of metastatic bone disease in the US population is not well understood. We sought to estimate the current number of US adults with metastatic bone disease using two large administrative data sets.

Methods: Prevalence was estimated from a commercially insured cohort (ages 18-64 years, MarketScan database) and from a fee-for-service Medicare cohort (ages $\geq 65$ years, Medicare $5 \%$ database) with coverage on December 31, 2008, representing approximately two-thirds of the US population in each age group. We searched for claims-based evidence of metastatic bone disease from January 1, 2004, using a combination of relevant diagnosis and treatment codes. The number of cases in the US adult population was extrapolated from age- and sexspecific prevalence estimated in these cohorts. Results are presented for all cancers combined and separately for primary breast, prostate, and lung cancer.

Results: In the commercially insured cohort (mean age $=42.3$ years [SD $=13.1]$ ), we identified 9505 patients $(0.052 \%)$ with metastatic bone disease. Breast cancer was the most common primary tumor type $(\mathrm{n}=4041)$. In the Medicare cohort (mean age $=75.6$ years $[\mathrm{SD}=7.8])$, we identified $6427(0.495 \%)$ patients with metastatic bone disease. Breast $(n=1798)$ and prostate $(n=1862)$ cancers were the most common primary tumor types. We estimate that $279,679(95 \%$ confidence interval: 274,579-284,780) US adults alive on December 31, 2008, had evidence of metastatic bone disease in the previous 5 years. Breast, prostate, and lung cancers accounted for $68 \%$ of these cases.

Conclusion: Our findings suggest that approximately 280,000 US adults were living with metastatic bone disease on December 31, 2008. This likely underestimates the true frequency; not all cases of metastatic bone disease are diagnosed, and some diagnosed cases might lack documentation in claims data.

Keywords: bone neoplasms, epidemiology, metastasis, prevalence

\section{Introduction}

Bone is a common site of metastatic cancer. ${ }^{1,2}$ Bone metastases occur in most tumor types but are most prevalent in cancers of the breast, prostate, and lung. ${ }^{3}$ These bone lesions can cause serious skeletal complications, including spinal cord or nerve root compression, bone surgery, hypercalcemia of malignancy, pathologic fractures, and severe bone pain requiring palliative radiotherapy, ${ }^{4,5}$ all of which can significantly compromise quality of life ${ }^{6-10}$ and may negatively affect survival. ${ }^{11,12}$ Palliation of pain, prevention of skeletal complications, and maintenance of quality of life are the primary objectives in managing patients with metastatic bone disease. ${ }^{5}$

A diagnosis of malignant bone disease generally represents an incurable cancer. However, the clinical course can be prolonged. Based on recent research using 
population-based and medical registries in Denmark, 1- and 3 -year cumulative survival in breast cancer patients diagnosed with bone metastases was $59 \%$ and approximately $22 \%,{ }^{13}$ respectively. Similarly, 1 - and 3 -year cumulative survival in prostate cancer patients diagnosed with bone metastases was $47 \%$ and approximately $9 \%,{ }^{14}$ respectively. These data demonstrate that for some patients, metastatic bone disease is a chronic condition that must be carefully managed.

To better understand the burden of metastatic bone disease in the United States, current and reliable estimates of prevalent cases are important. Estimates of incidence rates and subsequent survival in a population-based setting are available, ${ }^{13-17}$ but current prevalence data are scant. We sought to estimate the current number of patients with metastatic bone disease in the US adult population by applying a claims-based definition of metastatic bone disease to two large administrative data sets.

\section{Methods}

\section{Data sources and study participants}

Two data sources were used in this analysis: the MarketScan database and the Medicare 5\% database. MarketScan is a large commercial claims and encounters database of specific health services records, demographics, and enrollment information for employees and their dependants selected from employer health insurance plans. It includes information on diagnoses, procedures performed and reimbursed by commercial insurance, and dates of service.

The Medicare $5 \%$ database is a random sample of all Medicare beneficiaries. It includes the annual denominator file, which contains information on demographics and enrollment in Medicare and managed care organizations (health maintenance organizations [HMO]) for each beneficiary; and the annual claims-based Standard Analytic Files, which contain Part A institutional and Part B physician/suppliers files. Claims-based files include information on diagnoses, procedures performed and reimbursed by Medicare, and dates of service.

Two point-prevalent study cohorts were assembled of beneficiaries with insurance coverage on December 31, 2008. The first included employees and their dependants enrolled in fee-for-service plans through their employers and aged 18-64 years on December 31, 2008 ("commercially insured" cohort). The second included Medicare Parts A and B beneficiaries not enrolled in HMOs ("fee-for-service Medicare" cohort), residing in the 50 states and District of Columbia and aged $\geq 65$ years on December 31,2008 . We examined all available claims for each cohort member in the preceding 5 years (January 1, 2004, through December 31, 2008) for evidence of metastatic bone disease.

\section{Identification of metastatic bone disease, cancer, and} cancer type

Patients were identified as having metastatic bone disease by (1) at least one inpatient claim or two outpatient claims on different days within any 12-month interval, carrying the International Classification of Diseases, Ninth Revision, Clinical Modification (ICD-9-CM) diagnosis code for secondary malignant neoplasm of bone or bone marrow (198.5); or (2) at least one claim carrying Healthcare Common Procedure Coding System codes for intravenous (IV) bisphosphonates zoledronic acid (Zometa, J3487) or pamidronate (J2430) with at least one qualifying code on the same claim. Qualifying codes included the ICD-9-CM diagnosis codes for primary cancer (see Table 1); metastatic cancer in lymph nodes (196.xx), respiratory and digestive systems (197.xx), and other sites including bone and bone marrow (198.xx); and $\mathrm{V}$ codes for encounters for antineoplastic chemotherapy (V58.11) and immunotherapy (V58.12). The earliest date of

Table I International Classification of Diseases, Ninth Edition, Clinical Modification diagnosis codes used to identify cancer types

\begin{tabular}{ll}
\hline Cancer types & Diagnosis codes \\
\hline Head and neck & $140-14999$ \\
Esophagus & $150-15099$ \\
Stomach and small intestine & $151-15299$ \\
Colon and rectum & $153-15489$ \\
Liver & $155-15529$ \\
Gallbladder & $156-15699$ \\
Pancreas & $157-15799$ \\
Retroperitoneum and peritoneum & $158-15899$ \\
Spleen & $159-15999$ \\
Lung & $162-16399$ \\
Other respiratory & $160-16199,164-16599$ \\
Bone & $170-17099$ \\
Connective and soft tissue & $171-17199$ \\
Melanoma & $172-17299$ \\
Female breast & $174-17499$ \\
Male breast & 1750 or 1759 \\
Sarcoma & $176-17699$ \\
Gynecologic & $179-18499$ \\
Prostate & 185 \\
Other genitourinary & $186-18999$ \\
Central nervous system & $190-19299$ \\
Endocrine & $193-19499$ \\
Non-Hodgkin's lymphoma & $200-20099,202-20299$ \\
Hodgkin's lymphoma & $201-20199$ \\
Multiple myeloma & $203-20380$ \\
Leukemia & $204-20891$ \\
III-defined & $195-19599,199-19999$ \\
\hline
\end{tabular}


bone metastasis code or qualifying IV bisphosphonate code was defined as the bone metastasis index date.

These patients were also required to have claims-based evidence of primary cancer during a 12-month interval from 6 months before to 6 months after the bone metastasis index date. Cancer type was defined by presence of the ICD-9-CM diagnosis code for a specific cancer type (Table 1) on at least one inpatient claim or two outpatient claims on different days in the 12-month interval. We included multiple myeloma and primary bone malignancies in our study because, while we recognize that these are intrinsic diseases of the bone, any malignancies with invasion of bone (bone metastases from solid tumors, multiple myeloma, etc) share substantial clinical consequences and may be indicated for and benefit from similar management. Further, we wanted our analysis to be consistent with other reports of the burden of metastatic bone disease ${ }^{18}$ for comparison purposes. Solid tumors that most frequently metastasize to bone (breast cancer [female only], prostate cancer, and lung cancer) were of interest in this study. When there was claimsbased evidence of more than one of these three cancer types, the following algorithm was applied to identify a single type: (1) if there were bisphosphonate claims, the cancer type coded most frequently on these claims was chosen; (2) if there were no bisphosphonate claims, the cancer type coded most frequently on all claims during the 12-month interval was chosen.

\section{Estimation of number of prevalent cases in the national commercially insured population and fee-for-service Medicare population}

The number of prevalent cases of metastatic bone disease in the national commercially insured population aged 18-64 years was extrapolated with person-level weights derived from the Medical Expenditure Panel Survey and provided by the data vendor. ${ }^{19}$ The number of prevalent cases in the national fee-for-service Medicare population aged $\geq 65$ years was extrapolated by multiplying observed estimates in the $5 \%$ sample by twenty.

\section{Estimation of the number of prevalent cases in the US adult population}

To account for US citizens not represented in the commercially insured or the Medicare populations, the number of prevalent cases estimated in these two data sources was extrapolated to the entire US adult population by applying age- and sex-specific prevalence estimates to the 2008 US Census population. ${ }^{20}$ Age- and sex-specific prevalence estimates were produced by extrapolating the observed estimates for each age (18-44, 45-64, 65-74, 75-84, and $\geq 85$ years) and sex combination to the commercially insured population aged 18-64 years and the fee-for-service Medicare population aged $\geq 65$ years. Multiplying the age- and sex-specific prevalence estimates by counts of US populations in each category produced the total number of cases in each category. Summing these age- and sex-specific cases produced an estimated total number of patients living with metastatic bone disease in the US adult population as of December 31, 2008. This analysis was conducted for all cancers combined and then repeated for breast, prostate, and lung cancers.

All analyses were conducted using SAS software (version 9.1; SAS Institute Inc, Cary, NC).

\section{Results}

\section{Demographic characteristics}

The commercially insured cohort included 18,218,583 enrollees who met the inclusion criteria; $52.4 \%$ were aged 18-44 years and $47.7 \%$ were men. The fee-for-service Medicare cohort included 1,297,538 beneficiaries who met the inclusion criteria; $50.6 \%$ were aged $65-74$ years, $34.5 \%$ were aged $75-84$ years, and $42.2 \%$ were men.

\section{Number of patients with metastatic bone disease in the study cohorts}

Table 2 presents the number of patients meeting the case definition for metastatic bone disease and the distribution of case definition sources in each study cohort. In the commercially insured cohort, we identified 9505 patients $(0.052 \%$ of the cohort) living with metastatic bone disease on December 31 , 2008. Of the solid tumors examined separately, breast cancer was the most common primary tumor type $(n=4041)$. In the Medicare cohort, we identified 6427 (0.495\%) patients with metastatic bone disease. Breast $(\mathrm{n}=1798)$ and prostate $(n=1862)$ cancers were the most common primary tumor types.

Compared with elderly patients, younger patients with metastatic bone disease were more often identified from drug codes only (commercially insured: $27.9 \%$, Medicare: $20.1 \%$ ), less frequently identified from the diagnosis codes only (commercially insured: $28.9 \%$, Medicare: $37.0 \%$ ), and about equally as often identified from both drug and diagnosis codes (commercially insured: $43.3 \%$, Medicare: $43.0 \%$ ). Case definition sources varied considerably across the three 
Table 2 Number of patients meeting the metastatic bone disease case definition in the commercially insured and Medicare $5 \%$ sample cohorts and case definition sources, all cancers and by specific cancer types

\begin{tabular}{|c|c|c|c|c|c|c|c|c|}
\hline \multirow[t]{3}{*}{ Cancer type } & \multicolumn{4}{|c|}{ Commercially insured cohort $(n=18,218,583)$} & \multicolumn{4}{|c|}{ Medicare 5\% sample cohort $(n=1,297,538)$} \\
\hline & \multirow[t]{2}{*}{ n (\%) } & \multicolumn{3}{|c|}{ Definition sources $^{a}$} & \multirow[t]{2}{*}{ n (\%) } & \multicolumn{3}{|c|}{ Definition sources $^{a}$} \\
\hline & & $\begin{array}{l}\text { Diagnosis } \\
\text { codes only }\end{array}$ & $\begin{array}{l}\text { Drug } \\
\text { codes only }\end{array}$ & Both & & $\begin{array}{l}\text { Diagnosis } \\
\text { codes only }\end{array}$ & $\begin{array}{l}\text { Drug } \\
\text { codes only }\end{array}$ & Both \\
\hline All cancers & $9505(0.052)$ & 28.9 & 27.9 & 43.3 & $6427(0.495)$ & 37.0 & 20.1 & 43.0 \\
\hline Female breast & $404 I(0.022)$ & 17.6 & 23.9 & 58.5 & $1798(0.139)$ & 27.2 & 18.5 & 54.3 \\
\hline Prostate & $776(0.004)$ & 31.6 & 15.7 & 52.7 & $1862(0.144)$ & 40.3 & 11.9 & 47.8 \\
\hline Lung & $1253(0.007)$ & 49.2 & 10.2 & 40.6 & $795(0.061)$ & 57.5 & 6.9 & 35.6 \\
\hline Other & $3435(0.019)$ & 34.1 & 41.7 & 24.3 & $1972(0.152)$ & 34.5 & 34.5 & 31.0 \\
\hline
\end{tabular}

Note: apercent of row total.

cancer types of interest; the proportion of cases identified by diagnosis code alone was highest for lung cancer and lowest for breast cancer.

\section{Estimated number of prevalent cases of metastatic bone disease in the national commercially insured population and fee-for-service Medicare population}

By applying the person-level weights, we estimated a total of 60,411 (95\% confidence interval [CI]: 59,134$61,689)$ patients living with metastatic bone disease on December 31, 2008, among 120.7 million commercially insured persons aged 18-64 years in the United States; patients with breast, prostate, or lung cancer accounted for $64 \%$ of prevalent cases of metastatic bone disease. Among the 26 million fee-for-service Medicare enrollees in the United States, 128,540 (95\% CI: 125,485-131,595) patients were living with metastatic bone disease; $69 \%$ of cases occurred in patients with the specified cancer types. Table 3 presents estimates of prevalent cases of metastatic bone disease in these two large components of the insured US adult population for all cancers and by the three specific types of interest.

\section{Estimated number of prevalent cases of metastatic bone disease in the US adult population}

Table 3 also presents estimates of prevalent cases of metastatic bone disease for the entire US adult population based on the age- and sex-specific prevalence estimates as shown in Table 4. We estimated that a total of 279,679 (95\% CI: 274,579-284,780) US adults alive on December 31, 2008, had evidence of metastatic bone disease in the previous 5 years. Of the three cancer types examined separately, female breast cancer was the most common primary cancer type, accounting for about one-third of total metastatic bone disease cases in the US adult population $(n=90,904 ; 95 \%$ CI: 88,095-93,714). Prostate cancer was the second most common ( $\mathrm{n}=62,841 ; 95 \% \mathrm{CI}$ : 60,253-65,429), and lung cancer was the least common of the specific cancer types examined ( $n=35,222 ; 95 \%$ CI: 33,415-37,030). These three cancer types combined accounted for $68 \%$ of total metastatic bone disease cases.

\section{Discussion}

This descriptive study estimated the number of pointprevalent cases of metastatic bone disease in the US adult

Table 3 Estimated number of prevalent cases of metastatic bone disease in the national commercially insured population aged I8-64 years, the national fee-for-service Medicare population aged $\geq 65$ years, and the US adult population on December 31, 2008, all cancers and by specific cancer types

\begin{tabular}{|c|c|c|c|}
\hline Cancer type & $\begin{array}{l}\text { Commercially insured, } \\
\text { ages } 18-64 \text { years } \\
(n=\mid 20,694,145)\end{array}$ & $\begin{array}{l}\text { Fee-for-service Medicare, } \\
\text { ages } \geq 65 \text { years } \\
(n=25,950,760)\end{array}$ & $\begin{array}{l}\text { US adult population }^{a} \\
(n=230,1 \mid 8,000)\end{array}$ \\
\hline All cancers & $60,4 \mid I(59, \mid 34-6 I, 689)$ & $128,540(|25,485-| 3 \mid, 595)$ & $279,679(274,579-284,780$ \\
\hline Female breast & $25,754(24,9$ II-26,596) & $35,960(34,34 \mid-37,579)$ & $90,904(88,095-93,7 \mid 4)$ \\
\hline Prostate & $4,969(4,609-5,329)$ & $37,240(35,593-38,887)$ & 62,84 I $(60,253-65,429)$ \\
\hline Lung & $7,879(7,421-8,337)$ & $15,900(\mid 4,823-16,977)$ & $35,222(33,415-37,030)$ \\
\hline Other & $21,809(21,046-22,573)$ & $39,440(37,745-4 I, \mid 35)$ & $90,712(87,843-93,580)$ \\
\hline
\end{tabular}

Notes: Data presented as estimated number of patients with metastatic bone disease ( $95 \%$ confidence interval). ${ }^{a}$ US Census 2008. 
Table 4 Age- and sex-specific prevalence estimates of metastatic bone disease and distribution of age and sex in the US adult population

\begin{tabular}{|c|c|c|c|c|c|c|c|c|}
\hline \multirow[t]{2}{*}{ Sex } & \multirow[t]{2}{*}{ Age, years } & \multirow[t]{2}{*}{$\begin{array}{l}\text { Insured } \\
\text { population, }{ }^{\text {a }}\end{array}$} & \multicolumn{5}{|c|}{$\begin{array}{l}\text { Prevalence of metastatic bone disease (standard error), per } 10,000 \\
\text { population }\end{array}$} & \multirow[t]{2}{*}{$\begin{array}{l}\text { US } \\
\text { population, }{ }^{\mathrm{b}} \mathbf{n}\end{array}$} \\
\hline & & & All cancers & Female breast & Prostate & Lung & Other & \\
\hline \multirow[t]{5}{*}{ Men } & $18-44$ & $32,978,252$ & $0.64(0.04)$ & & $0.03(0.01)$ & $0.09(0.02)$ & $0.52(0.04)$ & $57,522,000$ \\
\hline & $45-64$ & $26,686,875$ & $6.96(0.14)$ & & I.82 (0.07) & I.35 (0.06) & $3.79(0.11)$ & $38,104,000$ \\
\hline & $65-74$ & $6,043,760$ & $41.99(1.18)$ & & $19.33(0.80)$ & $7.05(0.48)$ & $15.62(0.72)$ & $9,265,000$ \\
\hline & $75-84$ & $3,686,380$ & 73.41 (1.99) & & $45.74(\mathrm{I} .57)$ & $8.30(0.67)$ & 19.37 (I.02) & $5,336,000$ \\
\hline & $\geq 85$ & $1,210,060$ & $90.74(3.86)$ & & $71.90(3.43)$ & $4.30(0.84)$ & I4.54 (I.55) & $1,864,000$ \\
\hline \multirow[t]{5}{*}{ Women } & $18-44$ & $32,729,023$ & I.4I (0.06) & $0.99(0.05)$ & & $0.10(0.02)$ & $0.33(0.03)$ & $55,666,000$ \\
\hline & $45-64$ & $28,299,994$ & $12.40(0.19)$ & $7.96(0.15)$ & & $1.29(0.06)$ & $3.15(0.10)$ & $39,956,000$ \\
\hline & $65-74$ & $7,076,540$ & 40.39 (1.07) & $22.53(0.80)$ & & $6.25(0.42)$ & I I.62 (0.57) & $10,858,000$ \\
\hline & $75-84$ & $5,269,160$ & $50.63(1.38)$ & $27.40(1.02)$ & & $5.43(0.45)$ & $17.80(0.82)$ & $7,689,000$ \\
\hline & $\geq 85$ & $2,664,860$ & $37.00(1.66)$ & $20.94(1.25)$ & & $2.93(0.47)$ & 13.13 (0.99) & $3,858,000$ \\
\hline
\end{tabular}

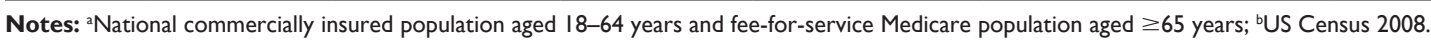

population on December 31, 2008, using a large commercial insurance claims database and the Medicare 5\% sample database. Our findings suggest that approximately 280,000 US adults were living with metastatic bone disease on December 31,2008 , with $68 \%$ of cases occurring in patients with primary breast, prostate, or lung cancer.

To our knowledge, Schulman and Kohles ${ }^{18}$ published the only other study aimed at describing the burden of metastatic bone disease in the US population in terms of prevalence. The similar magnitude of our national pointprevalence estimate $(279,679)$ and the Schulman and Kohles ${ }^{18}$ period-prevalence estimate of the size of the US population with metastatic bone disease between 2000-2004 $(256,137)$ obscures important differences in numbers by cancer type. Compared with their estimates, ours were $30 \%$ higher for breast cancer, $32 \%$ higher for prostate cancer, $5 \%$ lower for lung cancer, and $11 \%$ lower for all other cancer types combined. The study methodologies differ in important ways beyond estimation of point- versus period-prevalence. Schulman and Kohles ${ }^{18}$ included pediatric patients (though few cases were identified) and used prevalence estimates for Medicare enrollees with supplemental employer-paid insurance to extrapolate to the full Medicare population. How well this Medicare subset represents the entire Medicare population is debatable. We limited our extrapolation to the Medicare fee-for-service population, having studied a random sample of this population in the Medicare 5\% data set. Rather than make the assumption that the overall prevalence of metastatic bone disease in the population not covered by commercial insurance or by Medicare was the same as in the commercially insured or Medicare population, we took the approach of calculating observed age- and sex-specific prevalence, based on our extrapolation to the commercially insured (ages 18-64 years) and fee-for-service Medicare (aged $\geq 65$ years) populations, and applied these estimates to counts of the US adult population in each stratum from the US Census.

Another key difference is that we included bisphosphonate treatment in the case definition of bone metastasis. Schulman and Kohles ${ }^{18}$ identified patients with the first documented diagnosis of primary cancer, and followed claims over time for evidence of metastatic bone disease defined by at least one ICD-9-CM code for metastatic bone disease (198.5) or by a primary diagnosis code for a cancer type other than bone cancer and a secondary diagnosis code for bone cancer (170.xx). We assessed whether cohort members met a claimsbased definition for metastatic bone disease before identifying cancer type. Our case definition required one inpatient claim with an ICD-9-CM code of 198.5 or two outpatient claims with this code on different days within any 1-year interval between 2004-2008 (to avoid including patients who were tested to rule out metastatic bone disease and had no further claims evidence of metastatic bone disease). We also included in the case definition one or more claims for IV zoledronic acid (Zometa) or pamidronate, provided the claim included a code for cancer or cancer treatment. The only licensed indications for these two therapies in cancer patients are bone lesions from solid tumors or multiple myeloma and hypercalcemia of malignancy, which is relatively rare and even rarer in the absence of bone metastasis. ${ }^{21}$ Our decision to include bisphosphonate treatment in the case definition was motivated by concern that use of diagnosis codes alone to identify metastatic bone disease results in relatively low sensitivity. ${ }^{22}$

The strength of this study is that the data sets used included representative samples of two large components of the US adult population. The commercial claims database is 
one of the larger in the US and contains data on approximately 24 million employees and their dependants aged younger than 65 years who have health insurance through their employers. Approximately 18 million individuals were included in our study. Weights contained in this database allowed us to project estimates of bone metastasis prevalence to approximately 121 million US adults aged 18-64 years covered by employer-sponsored insurance, representing $63 \%$ of the total US population of this age range. The Medicare 5\% sample database is essentially a random sample of approximately 44 million Medicare beneficiaries. Estimates from approximately 1.3 million individuals eligible for our study were projected to 26 million Medicare fee-for-service enrollees with Part A and Part B coverage, accounting for two-thirds of the US population aged $\geq 65$ years. Furthermore, age and sex distribution in the two projected populations were similar to the total US adult population aged $18-64$ and $\geq 65$ years, respectively.

Several limitations should be noted when interpreting our findings. While we examined claims from 2004-2008 for evidence of metastatic bone disease, estimates of prevalence in this study cannot be properly interpreted as measures of the prevalence of 5-year history of metastatic bone disease, because not all individuals included in this study were continuously insured within the data sets studied. Of the commercially insured enrollees, $83.4 \%, 46.0 \%, 21.6 \%, 14.6 \%$, and $11.1 \%$ had at least 1 year, 2 years, 3 years, 4 years, and 5 years of continuous coverage, respectively. The corresponding distribution of the fee-for-service Medicare enrollees was $93.3 \%, 86.1 \%$, $79.7 \%, 74.2 \%$, and $68.9 \%$. Patients with relatively shorter duration of insurance coverage without claims evidence for metastatic bone disease may have had evidence of metastatic bone disease during the period without insurance coverage. The probable effect of examining less than 5 years of claims is underestimation of prevalence, as persons may be falsely identified as not having a history of metastatic bone disease. To explore this issue, we performed a post hoc analysis. We estimated separately in each cohort the crude probability of de novo evidence of metastatic bone disease in calendar year $Y$, given no evidence of metastatic bone disease from $\mathrm{Y}+1$ to 2008 and continuous enrollment from $\mathrm{Y}$ to 2008 , for $\mathrm{Y}=2004,2005$, 2006, and 2007. We used these probabilities to inflate prevalence estimates, such that the modified estimates could be interpreted as (extrapolated) measures of the prevalence of 5-year history of metastatic bone disease. We found that the number of persons with a 5-year history of metastatic bone disease could be as high as 322,180 (versus 279,679 in Table 3) US adults. These modified estimates may be high, as the most common causes of non-continuous enrollment - a recent employment change in the non-elderly and a recent 65 th birthday in the elderly - likely predict the absence of metastatic bone disease.

Second, not all diagnoses of metastatic bone disease result in corresponding claims codes. Few studies have examined the validity of using diagnosis codes to identify metastatic bone disease. The only such study we are aware of was done in Denmark and evaluated use of the ICD-10 code for metastatic bone disease (C79.5). ${ }^{22}$ Using the National Registry of Patients database and data from medical chart reviews as the gold standard, Jensen et $\mathrm{a}^{22}$ found relatively low sensitivity (0.44 [95\% CI: $0.30-0.60$ ] for prostate cancer and 0.32 [95\% CI: 0.13-0.57] for breast cancer) and very high specificity. These results may not be applicable to the US experience. The ICD-10 diagnosis code set is substantially different in structure and concept from the ICD-9 code set, which is still in use in the United States. In addition, the coding practices may differ. Our use of codes for IV bisphosphonates relevant to treatment of metastatic bone disease to supplement the diagnosis code should help improve sensitivity, but this also presents limitations. For example, patients with kidney dysfunction or known risk factors for osteonecrosis of the jaw may not be candidates for bisphosphonate therapy. Additionally, patients who underwent orthopedic procedures or palliative radiation for a malignant fracture may not be identified by the codes used. Ultimately, the sensitivity and specificity of our claimsbased definition of metastatic bone disease remains unknown until a validation study is performed.

Third, the age- and sex-specific prevalence estimates we applied to the US Census counts in each stratum were based on results from analysis of the commercially insured and feefor-service Medicare populations. Prevalence of metastatic bone disease in the remainder of the adult population may differ from prevalence in the commercially insured and feefor-service Medicare populations. Remaining subgroups of US adults include people with private insurance, uninsured people, Medicaid beneficiaries without Medicare coverage, Medicare Advantage participants, and military forces. The commercially insured and fee-for-service Medicare populations account for approximately $60 \%$ of the US adult population; the impact of possible differential prevalence in other subgroups is unclear.

A last important consideration in interpreting our estimates is that not all cases of metastatic bone disease are diagnosed. Patients with a poor prognosis at the time of cancer diagnosis might not receive tests for metastatic bone disease, with the rationale that they will die soon and 
the information will not change treatment decisions. Other patients might develop asymptomatic metastatic bone disease and live with it for some time before it becomes symptomatic or is discovered during routine testing. This study reports only cases of metastatic bone disease that have come to the attention of health care providers and were recorded.

Despite these limitations, our data provide a recent perspective on prevalence of metastatic bone disease and increase knowledge about the distribution across cancer types.

\section{Acknowledgments and disclosure}

This study was supported by a research contract from Amgen Inc, Thousand Oaks, California. The contract ensured Minneapolis Medical Research Foundation authors had final determination of the content of this manuscript. SL, YP, EDW, and TJA are employed by the Chronic Disease Research Group. KC, VMC, SS, JJP, and JFA are employed by Amgen Inc. AHB reports no conflicts of interest. The authors thank Chronic Disease Research Group colleagues Shane Nygaard for manuscript preparation and Nan Booth, MSW, MPH, ELS, for manuscript editing.

\section{References}

1. Roodman GD. Mechanisms of bone metastasis. $N$ Engl J Med. 2004;350(16):1655-1664.

2. Coleman RE. Metastatic bone disease: clinical features, pathophysiology and treatment strategies. Cancer Treat Rev. 2001;27(3):165-176.

3. Coleman RE. Bisphosphonates: clinical experience. Oncologist. 2004;9(Suppl 4):14-27.

4. Coleman RE. Clinical features of metastatic bone disease and risk of skeletal morbidity. Clin Cancer Res. 2006;12(20 Pt 2):6243-6249.

5. Selvaggi G, Scagliotti GV. Management of bone metastases in cancer: a review. Crit Rev Oncol Hematol. 2005;56(3):365-378.

6. Lipton A. Management of bone metastases in breast cancer. Curr Treat Options Oncol. 2005;6(2):161-171.

7. Clemons MJ, Dranitsaris G, Ooi WS, et al. Phase II trial evaluating the palliative benefit of second-line zoledronic acid in breast cancer patients with either a skeletal-related event or progressive bone metastases despite first-line bisphosphonate therapy. J Clin Oncol. 2006;24(30): 4895-4900.

8. Wardley A, Davidson N, Barrett-Lee P, et al. Zoledronic acid significantly improves pain scores and quality of life in breast cancer patients with bone metastases: a randomised, crossover study of community vs hospital bisphosphonate administration. Br J Cancer. 2005;92(10):1869-1876.
9. Weinfurt KP, Li Y, Castel LD, Timbie JW, Glendenning GA, Schulman KA. The impact of skeletal-related events on health-related quality of life of patients with metastatic prostate cancer [abstract 662P]. Ann Oncol. 2002;13(Suppl 5):180.

10. Weinfurt KP, Castel LD, Li Y, Timbie JW, Glendenning GA, Schulman KA. Health-related quality of life among patients with breast cancer receiving zoledronic acid or pamidronate disodium for metastatic bone lesions. Med Care. 2004;42(2):164-175.

11. Oefelein MG, Ricchiuti V, Conrad W, Resnick MI. Skeletal fractures negatively correlate with overall survival in men with prostate cancer. J Urol. 2002;168(3):1005-1007.

12. Saad F, Lipton A, Cook R, Chen YM, Smith M, Coleman R. Pathologic fractures correlate with reduced survival in patients with malignant bone disease. Cancer. 2007;110(8):1860-1867.

13. Yong M, Jensen AÖ, Jacobsen JB, Nørgaard M, Fryzek JP, Sørensen HT. Survival in breast cancer patients with bone metastases and skeletal-related events: a population-based cohort study in Denmark (1999-2007). Breast Cancer Res Treat. 2011;129(2):495-503.

14. Nørgaard M, Jensen AØ, Jacobsen JB, Cetin K, Fryzek JP, Sørensen HT. Skeletal related events, bone metastasis and survival of prostate cancer: a population based cohort study in Denmark (1999 to 2007). J Urol. 2010;184(1):162-167.

15. Chia VM, Cetin K, Jacobsen JB, et al. The incidence and prognostic significance of bone metastases and skeletal-related events in lung cancer patients: a population-based cohort study in Denmark [Meeting Abstracts]. J Clin Oncol. 2010;28(15 Suppl e18074).

16. Sathiakumar N, Delzell E, Morrisey MA, et al. Mortality following bone metastasis and skeletal-related events among women with breast cancer: a population-based analysis of US Medicare beneficiaries, 1999-2006. Breast Cancer Res Treat. 2012;131(1):231-238.

17. Sathiakumar N, Delzell E, Morrisey MA, et al. Mortality following bone metastasis and skeletal-related events among men with prostate cancer: a population-based analysis of US Medicare beneficiaries, 1999-2006. Prostate Cancer Prostatic Dis. 2011;14(2):177-183.

18. Schulman KL, Kohles J. Economic burden of metastatic bone disease in the US. Cancer. 2007;109(11):2334-2342.

19. Sommers J. Updates to the Medical Expenditure Panel Survey Insurance Component List Sample Design, 2004. Methodology Report No 18. Available from: http://www.meps.ahrq.gov/mepsweb/ data_files/publications/mr18/mr18.pdf. Jan 2007. Accessed Jan 12 , 2012.

20. US Census Bureau. Resident population by sex and age: 1980 to 2008. Available from: http://www.census.gov/compendia/statab/2010/ tables/10s0007.pdf. Accessed January 12, 2012

21. Vassilopoulou-Sellin R, Newman BM, Taylor SH, Guinee VF. Incidence of hypercalcemia in patients with malignancy referred to a comprehensive cancer center. Cancer. 1993;71(4):1309-1312.

22. Jensen AØ, Nørgaard M, Yong M, Fryzek JP, Sørensen HT. Validity of the recorded International Classification of Diseases, 10th edition diagnoses codes of bone metastases and skeletal-related events in breast and prostate cancer patients in the Danish National Registry of Patients. Clin Epidemiol. 2009;1:101-108.
Clinical Epidemiology

\section{Publish your work in this journal}

Clinical Epidemiology is an international, peer-reviewed, open access journal focusing on disease and drug epidemiology, identification of risk factors and screening procedures to develop optimal preventative initiatives and programs. Specific topics include: diagnosis, prognosis, treatment, screening, prevention, risk factor modification, systematic

\section{Dovepress}

reviews, risk \& safety of medical interventions, epidemiology \& biostatical methods, evaluation of guidelines, translational medicine, health policies \& economic evaluations. The manuscript management system is completely online and includes a very quick and fair peer-review system, which is all easy to use. 